PROTOCOLO DE CONDUTA

\title{
Cuidados perioperatórios em transplante de pâncreas
}

\section{Perioperative care in pancreatic transplantation}

Bruno Barbosa Papaléo Filho ${ }^{1}$. Flávio Lobo Maia' ${ }^{1,2}$. Marcelo Lima Mont’Alverne Rangel ${ }^{1,2}$. Josiane dos Santos Garcez ${ }^{1}$. Lia Cavalcante Cezar ${ }^{1,2}$.

1 Universidade Federal do Ceará (UFC), Fortaleza, Ceará, Brasil. 2 Hospital Universitário Walter Cantídio (HUWC), Fortaleza, Ceará, Brasil.

\section{RESUMO}

Diabéticos insulinodependentes são susceptíveis a altas taxas de complicações secundárias. O transplante de pâncreas teve início na Universidade de Minesota em 1966, com altas taxas de insucessos. Porém, recentemente, este procedimento tem obtido melhores resultados, conjuntamente com outros transplantes de órgãos. Neste trabalho serão abordadas etiologia, fisiopatologia, técnica cirúrgica e as condutas referentes ao manejo anestésico pré, intra e pós-operatório no receptor de transplante de pâncreas. Objetivo: Abordar de forma objetiva o transplante de pâncreas, suas indicações e modalidades, tendo como foco o manejo anestésico perioperatório e uniformizar suas condutas. Método: Pesquisa bibliográfica nos sites periódicos Scielo, Pubmed, BVS e Lilacs referente às palavras-chave, assim como na literatura clássica atual. Resultado: Maior uniformização nas condutas anestésicas e fornecimento de uma fonte objetiva de pesquisa e protocolo de condutas direcionado para a prática da anestesia no transplante de pâncreas. Conclusão: A anestesia no transplante de pâncreas necessita experiência, habilidades técnicas e conhecimentos específicos para o seu correto manejo, visando o melhor prognóstico para o paciente.

Palavras-chave: Transplante. Anestesia. Pâncreas. Diabetes Mellitus.

\begin{abstract}
Insulin dependent diabetics are susceptible to high rates of secondary complications. Pancreas transplantation began at the University of Minnesota in 1966, with high failure rates. However, recently, this procedure has obtained better results, in conjunction with other organ transplants. In this paper, the etiology, pathophysiology, risk factors, surgical technique and the management of pre, intra and postoperative anesthetic management in the recipient of pancreas transplantation will be addressed. Objective: Objectively approach the transplantation of pancreas, its indications and modalities, focusing on perioperative anesthetic management and standardizing its conducts. Method: Bibliographic search in Scielo, Pubmed, BVS and Lilacs periodical sites for keywords, as well as in current classical literature. Result: Greater uniformity in anesthetic conducts and supply of an objective source of research and protocol of conducts directed to the practice of anesthesia in the transplantation of pancreas. Conclusion: Anesthesia in pancreas transplantation requires experience, technical skills and specific knowledge for its correct management, aiming at the best prognosis for the patient.
\end{abstract}

Keywords: Transplantation. Anesthesia. Pancreas. Diabetes Mellitus.

Autor correspondente: Bruno Barbosa Papaléo Filho, Travessa Hilnete, número 99, Dionísio Torres, Fortaleza, Ceará. CEP: 60135-210. Telefone: +55 85 99982-3014. E-mail: brunofilho.barbosa@hotmail.com

Conflito de interesses: Não há qualquer conflito de interesses por parte de qualquer um dos autores.

Recebido em: 05 Jan 2018; Revisado em: 19 Mar 2018; Aceito em: 23 Abr 2019. 


\section{INTRODUÇÃO}

Kelly e Lillehei, da Universidade de Minesota, realizaram o primeiro transplante de pâncreas em humanos, com ligadura do ducto pancreático para controle da secreção exócrina do pâncreas e muitas técnicas diferentes foram descritas nas duas décadas seguintes. O objetivo do transplante de pâncreas é melhorar a qualidade de vida dos pacientes diabéticos tipo 1 , retardando ou mesmo, impedindo a progressão das complicações do diabetes mellitus (DM), a fim de estabelecer um estado de normoglicemia insulino-independente. Vários estudos indicam que apenas o controle rigoroso da glicemia previne efetivamente a progressão da retinopatia, nefropatia, polineuropatia e vasculopatia diabética. ${ }^{1}$

Existem 3 categorias de transplante de pâncreas:

1. O Transplante de Pâncreas Isolado (TPA) é indicado para pacientes diabéticos com hiperlabilidade, que se apresentam em coma tanto por hiperglicemia como por hipoglicemia;

2. Transplante de Pâncreas e Rim Simultâneo (SPK) é indicado para pacientes diabéticos e nefropatas em diálise, com insuficiência renal crônica;

3. Transplante de Pâncreas após Transplante de Rim (PAK) é indicado para pacientes diabéticos que têm deterioração do enxerto renal por piora do diabetes. ${ }^{1}$

Geralmente, o pâncreas inteiro é transplantado de doadores falecidos. Mais raramente, o pâncreas distal é transplantado de um doador vivo. ${ }^{2}$

\section{OBJETIVO}

Abordar de forma objetiva o transplante de pâncreas, suas indicações e modalidades, tendo como foco o manejo anestésico perioperatório e uniformizar suas condutas.

\section{METODOLOGIA}

Trata-se de um estudo bibliográfico, com pesquisa de dados publicados em periódicos na base de dados Scielo, Pubmed, BVS e Lilacs. A partir dos artigos obtidos foi selecionado os de maior relevância sobre o tema abordado.

\section{INDICAÇÕES PARA O TRANSPLANTE DE PÂNCREAS E RIM-PÂNCREAS}

O transplante de pâncreas permite uma oferta de insulina endógena para pacientes com DM insulinodependente.

Os transplantes SPK e PAK são indicados em pacientes com diabetes e insuficiência renal crônica (IRC) que são considerados candidatos apropriados para transplante renal ou que já foram submetidos a transplante renal.

A PTA (pancreas transplant alone) é indicada em pacientes com diabetes que não necessitam de transplante renal e têm história de complicações metabólicas frequentes e graves ou problemas para manter a terapia com insulina, ocasionando complicações diabéticas intratáveis.

A maior parte dos pacientes submetidos ao transplante de pâncreas tem diabetes mellitus tipo 1. As indicações raras para o transplante de pâncreas são casos selecionados de diabetes mellitus tipo 2, pancreatite crônica com deficiência endócrina, fibrose cística com deficiência endócrina e pancreatectomia total anterior.

Entre os pacientes com diabetes e IRC, os menores de 50 anos têm uma sobrevida melhor do que os pacientes idosos quando submetidos a transplante de pâncreas simultâneo com transplante renal.

$\mathrm{O}$ aumento na sobrevivência na SPK pode ser devido à diminuição nas complicações de doença arterial coronariana (DAC). Para os pacientes com função renal normal que são submetidos ao TPA, a sobrevivência a longo prazo parece ser a mesma que em pacientes que recebem terapia com insulina crônica. ${ }^{3}$

\section{FISIOPATOLOGIA DA INSUFICIÊNCIA PANCREÁTICA}

O diabetes mellitus tipo 1 desenvolve-se consequentemente à destruição de células beta das ilhotas pancreáticas ocasionando uma perda permanente da produção endógena de insulina, necessitando de terapia de insulina exógena ao longo da vida. A causa subjacente do diabetes mellitus tipo 1 permanece desconhecida. O diabetes mellitus tipo 2 resulta da resistência periférica aos efeitos da insulina. Ambas as doenças produzem aumentos das concentrações de glicose no sangue, resultando em manifestações multiorgânicas do diabetes. Doença coronariana, doença cerebrovascular e doença vascular periférica ocorrem em pacientes com DM devido à aceleração da aterosclerose. Os pacientes com diabetes desenvolvem DAC mais cedo, são mais propensos a ter sintomas atípicos e têm uma maior taxa de mortalidade de infarto agudo do miocárdio (IAM) do que os não diabéticos.

Neuropatias periféricas e autonômicas se desenvolvem devido à DM, ocasionando em gastroparesia, parestesia nas extremidades inferiores, ulcerações, hipotensão ortostática e labilidade hemodinâmica. Os pacientes com diabetes apresentam alta prevalência de cegueira (16\%), insuficiência renal $(22 \%)$, amputação das extremidades inferiores (12\%), IAM (21\%) e acidente vascular cerebral (AVC) (10\%). As complicações agudas do DM tipo 1 tipicamente envolvem condições associadas a hiperglicemia grave, como cetoacidose diabética e coma hiperosmolar não-cetônico hiperglicêmico, enquanto a hipoglicemia é um resultado direto da administração de insulina exógena. ${ }^{3}$

\section{AVALIAÇÃO PRÉ-ANESTÉSICA E PREPARO PREOPERATÓRIO}

A avaliação pré-operatória para o paciente submetido a transplante de pâncreas envolve anamnese, exame físico e 
análise de todas as complicações crônicas e potenciais da DM tipo 1. Os centros de transplante de pâncreas realizam um processo abrangente e multidisciplinar de avaliação e seleção antes de listar os candidatos. Esta avaliação deve abordar os sistemas de órgãos mais afetados por diabetes de longa data, incluindo nefropatia, retinopatia, neuropatia e vasculopatias. $^{4}$

A avaliação da presença e gravidade da doença coronariana deve ser realizada em todos os candidatos, incluindo testes de isquemia não invasivos, avaliação da função ventricular e angiografia coronária em pacientes selecionados. ${ }^{3}$

Na maioria dos casos, o transplante de pâncreas envolve um órgão de doador falecido com um tempo de isquemia fria máximo de 24 horas; portanto, o transplante de pâncreas é considerado um procedimento urgente. A avaliação préoperatória realizada pelo anestesiologista deve se concentrar em qualquer alteração aguda do estado médico do paciente, especialmente aqueles que envolvem complicações diabéticas agudas, como cetoacidose e hipoglicemia. As medidas de glicemia e as administrações de insulina devem ser avaliadas rigorosamente antes da cirurgia. Avaliação da função renal em todos os candidatos é importante, pois a maioria dos pacientes com diabetes listados para o transplante de pâncreas também possui IRC e será submetido a transplante simultâneo de rim - pâncreas (SPK). Uma avaliação completa dos eletrólitos, incluindo a creatinina e o potássio, deve ser obtida antes da cirurgia, pois muitos pacientes são hipercalêmicos e toleram bem esta condição.

A avaliação seriada das últimas medidas de pressão arterial nos pacientes internados deve ser rigorosa, pois a maioria deles apresenta história de hipertensão importante com necessidade de uso de vários medicamentos, principalmente aqueles com IRC. A avaliação pré-operatória do estado do volume intravascular é especial em pacientes com IRC em hemodiálise, pois deve-se evitar tanto desidratação quanto hipervolemia. ${ }^{3}$

Finalmente, deve ser realizado um exame físico direcionado com foco na via aérea e no sistema cardiopulmonar. A incidência de dificuldade na intubação traqueal em pacientes com DM de longa data é verificada com frequência devido a alterações anatômicas da via aérea superior e dificuldade de posicionamento em hiperextensão, principalmente nos pacientes com artrite cervical e obesidade. ${ }^{3}$

É recomendado também incluir na avaliação clínica a checagem da tipagem sanguínea, reserva de hemoderivados e do regime de imunossupressão como a metilprednisona e a timoglobulina.

Esses pacientes diabéticos devem receber no período perioperatório soluções hidroeletrolíticas contendo frutose e medidas glicêmicas horárias.

Podem ser administrados benzodiazepínicos como medicação pré-anestésica. ${ }^{4}$ São recomendados procinéticos, que provocam efeito gastropropulsilvo associado ao aumento do tônus do esfíncter esofágico inferior e relaxamento da cárdia (metoclopramida $10 \mathrm{mg}$, ou bromoprida $10 \mathrm{mg}$ IV, IM ou VO 60- 90 minutos antes da indução) e antiácidos não particulados que atuam como tampão, reduzindo a acidez de qualquer conteúdo gástrico residual (citrato de sódio 30mL VO 1520 minutos antes da indução), pois os pacientes diabéticos e aqueles com IRC têm uma alta probabilidade de gastroparesia, aumentando o risco de aspiração de conteúdo gástrico. ${ }^{3}$

O paciente é admitido no hospital em jejum e tem início a preparação para o transplante, com a administração da Metilprednisolona (Solumedrol $\AA 500 \mathrm{mg}$ em SF 0,9\% $200 \mathrm{ml}$ em 2 horas), Micofenolato (Myfortic $\AA 360 \mathrm{mg} 1$ comprimido (cp) VO), Paracetamol 750mg 1 comprimido VO e Fenergan $\AA$ 25 mg 2 cp VO (medicação pré Thymoglobulina).

Antes da indução anestésica será administrado Omeprazol 40 mg EV, Unasyn ${ }^{\circledR}$ 3,5 g EV (que continuará por 48 horas), Fluconazol $400 \mathrm{mg}$ EV (continuará 200mg EV por 14 dias) e Thymoglobulina ${ }^{\circledR} 1,5 \mathrm{mg} / \mathrm{Kg} /$ dia (frasco de $25 \mathrm{mg}$ ) diluído em $500 \mathrm{ml}$ de SF 0,9\% em 6 horas que prosseguirá em dias alternados totalizando 4 doses. A profilaxia com heparina será iniciada após liberação da equipe cirúrgica na dose 5000 UI SC de $8 / 8 h .^{5}$

\section{MANEJO ANESTÉSICO INTRAOPERATÓRIO}

É necessária anestesia geral em todos os tipos de transplante de pâncreas, sendo recomendada intubação endotraqueal em sequência rápida com pressão cricoide contínua a fim de evitar broncoaspiração. Uma profundidade anestésica adequada e um bom relaxamento muscular são necessários para condições cirúrgicas ótimas. ${ }^{3}$

Deve ser obtido acesso venoso periférico e realizar monitorização com cardioscópio, pressão arterial invasiva e oximetria de pulso antes da indução anestésica, além da capnografia após intubação. ${ }^{3}$ Não se deve utilizar membros que contenham fistula arteriovenosa para o acesso venoso. ${ }^{4} \mathrm{~A}$ colocação da linha arterial antes da indução da anestesia pode ser considerada, especialmente em pacientes com hipertensão arterial sistêmica (HAS) grave ou DAC.

O monitoramento arterial é padrão para o transplante de pâncreas já que permite medições de pressão arterial contínua, bem como acesso para análise de gasometria arterial e monitoração de glicemia. ${ }^{3}$ No intraoperatório, deve-se manter a pressão arterial próxima de $120 \mathrm{X} 80 \mathrm{mmHg} .{ }^{5}$

Um acesso venoso central deve ser puncionado após indução anestésica e através dele serão administrados imunossupressores e drogas vasoativas. O monitoramento da pressão venosa central pode ser usado em alguns centros, no entanto, a utilidade desta prática foi questionada, uma vez que a pressão venosa central pode não ser um indicador confiável da capacidade de resposta a fluido intravascular. ${ }^{3}$

A indução anestésica pode ser feita com propofol ou etomidato e o relaxamento muscular com cisatracúrio. A Succinilcolina também pode ser utilizada, porém aumenta o risco de 
hipercalemia. Analgesia é feita principalmente com opioides (fentanil e/ou morfina). A manutenção da anestesia deve ser feita com Isoflurano em mistura de ar/oxigênio. ${ }^{4}$

$\mathrm{Na}$ indução anestésica, devem ser administrados como ATB profilático, Unasyn (3,5g EV), assim como Metilprednisolona $(0,5 \mathrm{mg} / \mathrm{kg})$. Após 30 minutos deve ser infundida a Thymoglobulina $(1,5 \mathrm{mg} / \mathrm{kg})$ em acesso central ou fístula artério - venosa em 6 horas. Recomenda-se o uso do Manitol $20 \%(0,7 \mathrm{~g} / \mathrm{Kg})$ infundido lentamente durante a anastomose do rim. $^{5}$

Pacientes com DM, IRC, doenças cardiovasculares e neuropatia autonômica podem ser propensos a grandes flutuações na frequência cardíaca e na pressão arterial. Os sinais vitais devem ser monitorados rigorosamente e a manutenção da estabilidade hemodinâmica deve ser um objetivo anestésico primário, especialmente durante e imediatamente após a indução anestésica.

Em pacientes com insuficiência renal, devem ser selecionados medicamentos que não apresentam eliminação renal. Todas as recomendações para o tratamento anestésico de receptores submetidos a transplante renal devem ser aplicadas para pacientes submetidos à transplante de rim - pâncreas simultâneo (SPK). ${ }^{3}$

Antes do desclampeamento das anastomoses vasculares, devese iniciar a ressuscitação volêmica adequada, pois a pré-carga cardíaca apropriada e as pressões arteriais normais devem ser os objetivos hemodinâmicos. Após o desbloqueio das conexões vasculares, manter a pressão de perfusão adequada ao enxerto é fundamental. A hipotensão deve ser corrigida rapidamente e a situação do volume intravascular deve ser otimizada. ${ }^{3}$

É sempre importante monitorizar temperatura com termômetro nasofaríngeo e utilizar dispositivos de aquecimento como colchão térmico, manta térmica e fluidos aquecidos. Em geral, objetiva-se manter Pressão Venosa Central (PVC) de aproximadamente $10 \mathrm{cmH}_{2} \mathrm{O}^{4}$

Deve-se utilizar preferencialmente solução SF 0,9\%, podendo também associar coloides, tratando episódios de hipotensão com reposição hídrica e evitando uso de vasoconstritor. Reposição sanguínea geralmente não é requerida. Durante a reperfusão do enxerto é fundamental evitar hipotensão. Após o término da técnica cirúrgica é feita a reversão do bloqueio neuromuscular e extubação. ${ }^{4}$

Um dos aspectos de cuidados intra-operatórios mais importantes para o transplante de pâncreas é o manejo da glicemia após a reperfusão do pâncreas. A monitorização glicêmica será inicialmente de $1 / 1$ hora, devendo - se manter a glicemia entre 100 e $150 \mathrm{mg} / \mathrm{dl}^{4}$

No esquema Ammon de insulina é o recomendado durante o transplante pancreático a preparação da solução com Insulina Regular 100U diluída em SF 0,9\% 100ml, alcançando-se a concentração de $1 \mathrm{U} / \mathrm{ml}$. Deve-se utilizar frascos de vidro para diminuir a perda de insulina por adsorção ao plástico e pela mesma razão deve-se desprezar os primeiros $10 \mathrm{ml} \mathrm{da}$ solução. A infusão da insulina é acompanhada da infusão de soro glicosado a 10\% (SG 10\%), ambos em bomba de infusão contínua (BIC), de acordo com a glicemia (mg/dl), da seguinte forma:

Quando a glicemia for menor que $70 \mathrm{mg} / \mathrm{dl}$, infunde-se SG $10 \%$ em BIC $75 \mathrm{ml} / \mathrm{h}$; se glicemia for entre 70 e $100 \mathrm{mg} / \mathrm{dl}$, infunde-se SG $10 \% 65 \mathrm{ml} / \mathrm{h}$; se glicemia entre 100 e $150 \mathrm{mg} /$ dl, inicia -se Insulina em BIC 1,5U/h e SG $10 \% 20 \mathrm{ml} / \mathrm{h}$; se glicemia entre 150 e 200, infunde-se Insulina $2 \mathrm{U} / \mathrm{h}$ e SG $10 \%$ $20 \mathrm{ml} / \mathrm{h}$; se glicemia entre 200 e $250 \mathrm{mg} / \mathrm{dl}$, infunde-se Insulina $3 \mathrm{U} / \mathrm{h}$ e SG $10 \% 20 \mathrm{ml} / \mathrm{h}$; se glicemia entre 250 e $300 \mathrm{mg} /$ dl, infunde-se Insulina 4U/h; se glicemia entre 300 e 400, infunde-se Insulina 6U/h (Tabela 1).

Gasometria arterial, eletrólitos, hemoglobina, hematócrito e glicemia devem ser coletados a cada hora. Durante a manipulação pancreática (anastomose e reperfusão) deve-se analisar a glicemia capilar a cada 30 minutos. $^{5}$

Após o desclampeamento, o pâncreas pode liberar insulina na circulação em vários minutos. A glicemia deve ser medida aproximadamente a cada 30 minutos para o restante do procedimento. Após um transplante bem-sucedido, a necessidade de insulina exógena diminui rapidamente, e os pacientes podem desenvolver hipoglicemia. $\mathrm{O}$ atraso na função do enxerto pode ser identificado pela presença de hiperglicemia. Neste caso, a infusão de insulina deve ser titulada para manter níveis de glicose no sangue inferiores a $200 \mathrm{mg} / \mathrm{dL}^{3}$.

Tabela 1. Algoritmo de infusão insulina-glicose (Glicemia Capilar, Insulina UI/h, SG $10 \% \mathrm{ml} / \mathrm{h}$ ).

\begin{tabular}{lcc}
\hline Glicemia capilar & Insulina Ul/h & SG $\mathbf{1 0} \% \mathbf{~ m l} / \mathbf{h}$ \\
\hline$<70$ & 0 & 75 \\
$70-100$ & 0 & 65 \\
$100-150$ & 1,5 & 20 \\
$150-200$ & 2 & 20 \\
$200-250$ & 3 & 20 \\
$250-300$ & 4 & 0 \\
$300-400$ & 6 & 0 \\
\hline
\end{tabular}

\section{TÉCNICA CIRÚRGICA}

A via de acesso normalmente usada é a laparotomia mediana. O implante do pâncreas é geralmente feito na fossa ilíaca direita do receptor, uma vez que os vasos ilíacos direitos são mais acessíveis. O implante pancreático pode ser feito de forma que a produção endócrina do órgão seja drenada pelo complexo venoso sistêmico ou portal.

As principais etapas do implante do enxerto pancreático são:

1. Incisão mediana iniciada de 2 a $5 \mathrm{~cm}$ acima da cicatriz umbilical e estendidas até o púbis; 
2. Exposição da bexiga, caso seja utilizada a derivação vesical de drenagem do conteúdo exócrino do pâncreas;

3. Mobilização, cranialmente, do ceco e do íleo terminal;

4. Exposição e mobilização dos vasos ilíacos (técnica semelhante à do transplante renal);

5. Anastomose término-lateral da veia porta do enxerto e a veia ilíaca comum do receptor (drenagem venosa sistêmica da secreção pancreática endócrina);

6. Anastomose término-lateral da artéria ilíaca comum do enxerto vascular em Y com a artéria ilíaca comum do receptor;

\section{Revascularização do enxerto (hemostasia);}

8. Drenagem da secreção exócrina pancreática do enxerto, que pode ser feita de duas maneiras:

8.1 Drenagem vesical com anastomose látero-lateral duodeno- vesical (modalidade realizada no HUWC);

8.2 Drenagem entérica (anastomose látero-lateral duodeno-jejunal ou em Y de Roux).

9. Revisão da hemostasia e lavagem da cavidade;

10. Fechamento da cavidade abdominal. ${ }^{3}$

\section{MANEJO PÓS-OPERATÓRIO}

Após o transplante de pâncreas, os pacientes devem ser monitorados na unidade de terapia intensiva (UTI). ${ }^{5}$ As medições regulares de glicemia devem ser continuadas no pós-operatório a fim de evitar hipoglicemia. Eletrólitos, hemograma completo e análise de gasometria arterial devem ser obtidos imediatamente após o procedimento, pois a anemia, os distúrbios eletrolíticos e ácido-base são comuns. Euvolemia deve ser mantida. Dependendo da idade do paciente e do risco subjacente para DAC, troponinas e eletrocardiogramas em série podem ser realizados para avaliar presença de isquemia miocárdica, pois os sintomas cardíacos podem ser ausentes nessa população.

A dor pós-operatória pode ser intensa devido à extensa ferida cirúrgica e, geralmente, é controlada com opioides no período intraoperatório com transição para analgesia controlada pelo paciente no pós-operatório precoce. A analgesia epidural pode

\section{REFERÊNCIAS}

1. Chaib E, Meirelles RF Jr, Santos VR, Bacchella T, D'Albuquerque LA. Transplante de pâncreas. Rev Med (São Paulo). 2009;88(3):13842.

2. Ferrari AT. Metodologia da pesquisa científica. São Paulo (SP): McGraw-Hill do Brasil; 1982.

3. Miller RD, Eriksson LI, Fleisher LA, Wiener-Kronish JP, Cohen NH, Young WL. Miller's anesthesia. 8. ed. Oxford (UK): Elsevier ser apropriada para receptores de transplante de pâncreas, embora a possibilidade de hipotensão no pós-operatório precoce possa ser desastrosa. ${ }^{3}$

Para transplante rim - pâncreas simultâneo, devem ser aplicadas as estratégias habituais de pós-operatório para pacientes com transplante de rim, incluindo monitoramento minucioso da diurese. ${ }^{3}$

\section{COMPLICAÇÕES}

As complicações ocorrem em 7\% a 9\% de todos os transplantes de pâncreas e normalmente requerem uma nova cirurgia. ${ }^{5} \mathrm{~A}$ trombose de enxerto é a complicação precoce mais importante e requer exploração cirúrgica de emergência. $\mathrm{O}$ sangramento intra-abdominal pode ocorrer secundariamente à coagulopatia induzida pela anticoagulação para o tratamento da trombose do enxerto. As principais complicações tardias incluem vazamento vesical ou entérico, sepse intra-abdominal e rejeição. ${ }^{3}$

Em 2008, houve 432 transplantes de pâncreas realizados nos Estados Unidos dos quais 223 foram TPA e 209 PAK, além dos 826 SPK. O número de transplantes de pâncreas realizados nos Estados Unidos para os três grupos diminuiu anualmente nos últimos anos. As razões para esse declínio não são bem

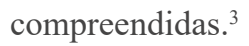

A sobrevida em 5 anos do paciente submetido a transplante de pâncreas é de $90 \%$ para PTA, $86 \%$ para PAK e $88 \%$ para SPK. A sobrevivência do enxerto em 5 anos para o transplante de pâncreas é de $73 \%$ para SPK, 52\% para TPA e $55 \%$ para PAK. ${ }^{3}$

Destaca-se a importância do processo de uniformização das condutas para transplante pâncreas, bem como a constante atualização e otimização das condutas baseadas em referências atuais, a fim de minimizar riscos ao paciente e prevenir complicações em consequência desse procedimento. ${ }^{6}$ É imperativo que os anestesiologistas tenham uma boa compreensão do manejo perioperatório desses pacientes para maximizar a capacidade de sobrevivência.

\section{CONCLUSÃO}

A modalidade de transplante mais realizada é o Transplante de Rim-Pâncreas simultâneo, sendo também o que promove melhores resultados quando a causa da IRC é o DM, garantindo a normoglicemia sem aumentar a morbimortalidade do transplante em Relação ao Transplante Renal isolado.

Health Sciences; 2015. Chapter 74, Anesthesia for abdominal organ transplantation, p.2270-2274.

4. Hospital Universitário Walter Cantídio. Protocolo de transplante de pâncreas do Hospital Universitário Walter Cantídio [Internet]. Fortaleza: HUWC; 2015 [Acesso em: 04 dez 2017]. Disponível em: http://www.ebserh.gov.br/web/huwc-ufc/protocolos-e-pops

5. Hospital Israelita Albert Einstein. Protocolo de preparo e 
acompanhamento do transplante de pâncreas - rim do Hospital Israelita Albert Einstein [Internet]. São Paulo; 2011 [Acesso em: 13 dez 2017]. Disponível em: http://bibliofarma.com/protocolo-depreparo-e-acompanhamento-do-transplante-de-pancreas-rim
6. Brasil. Ministério da Saúde. Portaria GM/MS no 1752, de 23 de setembro de 2005 [Internet]. Brasília: Ministério da saúde; 2005 [Acesso em: 10 dez 2017]. Disponível em: http://www.saude.mg.gov. br/atos_normativos/legislacao-sanitaria/estabelecimentos-de-saude/ transplantes-implantes/Portaria_1752.pdf

\section{Como citar:}

Papaléo BB Filho, Maia FL, Rangel ML, Garcez JS, Cezar LC. Cuidados perioperatórios em transplante de pâncreas. Rev Med UFC. 2019 outdez;59(4):87-92. 